\title{
Amorphous Se: A New Platform for Synthesizing Superparamagnetic Colloids with Controllable Surfaces
}

\author{
Unyong Jeong, Thurston Herricks, Edan Shahar, and Younan Xia* \\ Department of Chemistry, University of Washington, Seattle, Washington 98195-1700 \\ RECEIVED DATE xxx; E-mail: xia@chem.washington.edu
}

\section{Experimental Methods and Materials}

Synthesis of Colloids. All chemical reagents -- selenious acid $\left(\mathrm{H}_{2} \mathrm{SeO}_{3}, 99.99 \%\right.$, Aldrich), hydrazine hydrate $\left(\mathrm{N}_{2} \mathrm{H}_{4} \cdot \mathrm{xH}_{2} \mathrm{O}, 55 \% \quad \mathrm{~N}_{2} \mathrm{H}_{4}\right.$, Aldrich), and ethylene glycol (EG, 99.9\%, J. T. Baker) -- were used as received. $0.5 \mathrm{~mL}$ of iron oxide nanoparticles (EMG 305, Ferrotech, Nashua, NH) was dispersed in $40 \mathrm{~mL}$ EG and sonicated for $1 \mathrm{~h}$. The average diameter of the iron oxide particles was about $10 \mathrm{~nm}$. The iron oxide particles suspension in EG (1.0 mL) and the hydrazine hydrate solution in EG $(0.35 \mathrm{M}, 3 \mathrm{~mL})$ were added to $20 \mathrm{~mL}$ EG hosted in a 100 $\mathrm{mL}$ round bottom flask, stirred for $10 \mathrm{~min}$, and held at $-10{ }^{\circ} \mathrm{C} . \quad 6 \mathrm{~mL}$ pre-cooled selenious acid (0.07 $\mathrm{M}$, in EG) was introduced into the solution. The slow reduction rate at this temperature greatly promotes the role of iron oxide nanoparticles as nuclei. The light yellow color of the initial solution did not change at this stage. After $20 \mathrm{~min}$, the reaction vessel was quickly transferred into an oil bath heated at $50{ }^{\circ} \mathrm{C}$ and maintained for $10 \mathrm{~min}$. The color turned to light orange after about $6 \mathrm{~min}$ and deep orange after $10 \mathrm{~min}$. The vessel was finally placed in a water 
bath (held at $20{ }^{\circ} \mathrm{C}$ ) and the reaction was allowed to proceed for another $30 \mathrm{~min}$. The solution changed its color to red-orange. It is worth pointing out that the synthesis of $a$-Se colloids embedded with iron oxide nanoparticles required no additional surfactant or stabilizer other than those in the original ferrofluid.

Formation of $\mathrm{Pt}$ and $\mathrm{SiO}_{2}$ Coatings. Since $\mathrm{PtCl}_{2}$ (99.999\%, Aldrich) can also be readily reduced by hydrazine, all the residual hydrazine in the reaction solution was removed by vacuum distillation at room temperature. After $200 \mathrm{mg}$ poly(vinyl pyrrolidone) (PVP, Aldrich, $\mathrm{M}_{\mathrm{w}}=55,000$ ) had been introduced, the solution was heated to $62{ }^{\circ} \mathrm{C}$, and $50 \mathrm{mg} \mathrm{PtCl}_{2}$ was then added under vigorous magnetic stirring. When the solution became dark brown (usually after 3 $\mathrm{h}$ of reaction), $40 \mathrm{~mL}$ water was added and the product was centrifuged three times to completely remove excess PVP. For $\mathrm{SiO}_{2}$ coating, the Pt-coated colloids were redispersed in isopropyl alcohol $(20 \mathrm{~mL})$ hosted in a $50-\mathrm{mL}$ round bottom flask, followed by the addition of a small amount of PVP $(50 \mathrm{mg})$ and pure water $(5.0 \mathrm{~mL})$. Ammonium hydroxide (Fischer Scientific, $0.5 \mathrm{~mL}$ ) and tetraethyl orthosilicate $(99.999 \%$, Aldrich, $20 \mu \mathrm{L})$ were introduced successively. The reaction was allowed to proceed for $2 \mathrm{~h}$ and the final product was centrifuged twice with pure water.

Because the Pt coatings were polycrystalline, hydrazine could diffuse through the shells and dissolve the Se cores. In a typical procedure, the as-obtained product was centrifuged in a 1.5$\mathrm{mL}$ tube and water was decanted. $1 \mathrm{~mL} \mathrm{~N}_{2} \mathrm{H}_{4} \cdot \mathrm{xH}_{2} \mathrm{O}\left(55 \% \mathrm{~N}_{2} \mathrm{H}_{4}\right)$ was then introduced and sonication was applied for $20 \mathrm{sec}$ to re-disperse the colloids. The dark brown color of the suspension turned to orange within $5 \mathrm{~min}$ as Se was removed from the colloids. After $1 \mathrm{~h}$, the colloids were concentrated with a handy magnet and $\mathrm{N}_{2} \mathrm{H}_{4} \cdot \mathrm{xH}_{2} \mathrm{O}$ solution (light orange) was decanted. $1 \mathrm{~mL} \mathrm{~N}_{2} \mathrm{H}_{4} \cdot \mathrm{xH}_{2} \mathrm{O}$ was added for complete removal of Se. After 5 min, the colloids were collected using a handy magnet and washed with water three times and finally re-dispersed 
in water of an alcohol.

Loading of Iron Oxide Nanoparticles. From the EDX spectrum shown in Figure S3, the atomic percentage of $\mathrm{Fe}$ was estimated to be $\sim 2 \%$, which gives a volume fraction of $3.3 \%$ for the iron oxide nanoparticles (calculated in terms of $\mathrm{Fe}_{3} \mathrm{O}_{4}$ ) in the Pt-coated colloids after the Se cores had been removed.

\section{Immobilization of Alkanethiols and IgG}

16-mercaptohexadecanoic acid (90\%, Aldrich) and 1-hexadecanethiol (96\%, Research Chemicals Ltd.) were added to $5 \mathrm{~mL}$ ethanol to prepare a solution with a $4 \mathrm{mM}$ concentration for both thiols. This solution was then added to an ethanol suspension of the Pt-coated colloids (5 $\mathrm{mL}$ ) under magnetic stirring at room temperature. After $4 \mathrm{~h}$, the solution was centrifuged twice with pure water to wash out the excess thiols. The colloids were redispersed in pure water and an aqueous solution (1:5 molar ratio, $50 \mathrm{mM})$ of $\mathrm{N}$-hydroxysuccinimide $(98 \%$, Aldrich) and $\mathrm{N}$ (3-Dimethylaminopropyl)-N'-ethylcarbodiimide hydrochloride (commercial grade, Aldrich) was added. The reaction was kept in the dark for $3 \mathrm{~h}$ at room temperature. The excess chemicals were removed by centrifugation and washing twice with pure water. Anti-mouse IgG (Sigma) tagged with fluorescent dye -- fluorescein isothiocyanate (FITC), was added to the suspension. The IgG concentration in the solution was controlled at $2.5 \mu \mathrm{g} / \mathrm{mL}$. The reaction mixture was incubated in the dark for $3 \mathrm{~h}$ at room temperature, centrifuged, and re-dispersed in water. 

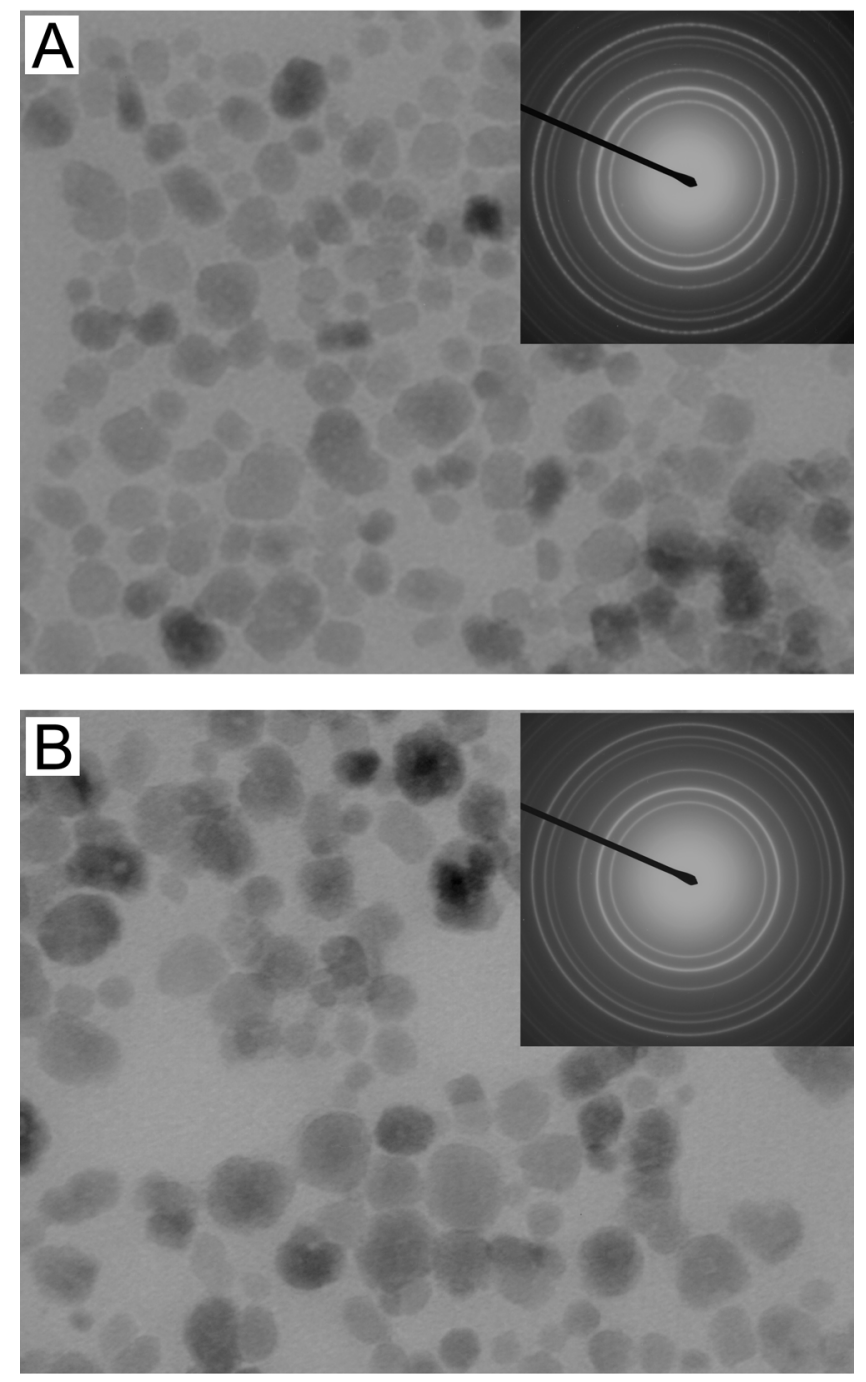

$20 \mathrm{~nm}$

Figure S1. TEM images of iron oxide nanoparticles and their electron diffraction patterns. (A) Nanoparticles contained in the commercial ferrofluid. (B) Nanoparticles after they had been collected from the ferrofluid and treated with hydrazine for $1 \mathrm{~h}$. There was essentially no change in morphology and crystal structure before and after hydrazine treatment. 


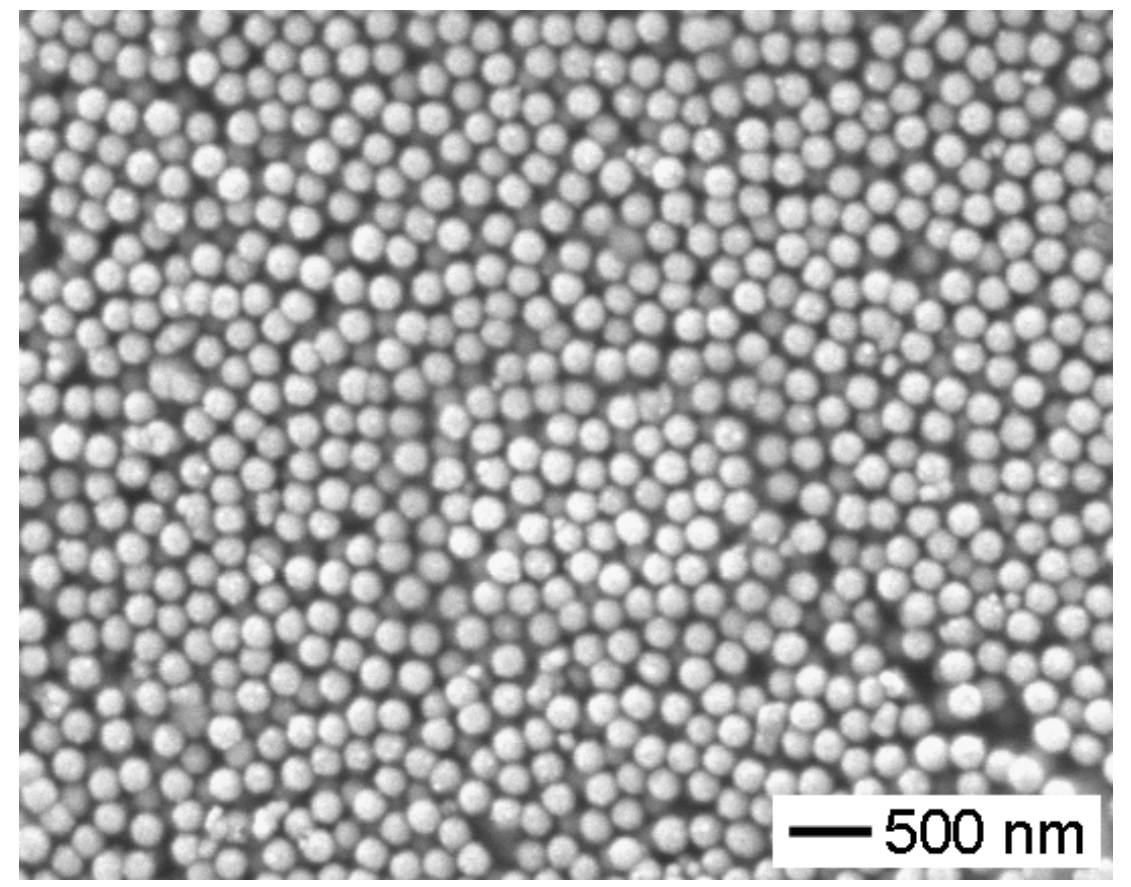

Figure S2. SEM image of the Pt shells obtained by selectively dissolving the $a$-Se cores with hydrazine. These hollow particles maintained their spherical shape and size monodispersity during the removal of Se. 


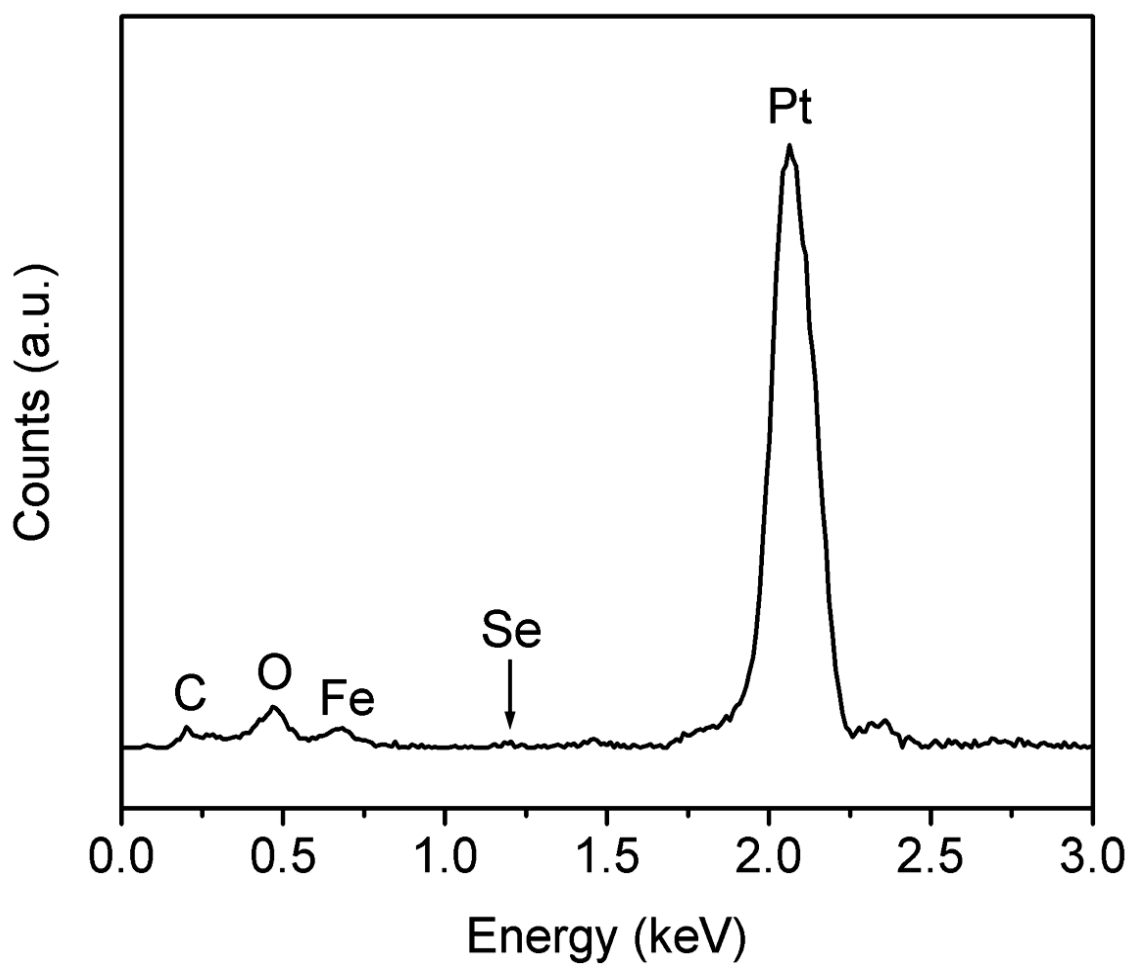

Figure S3. The EDX spectrum of a Se@Pt sample after the $a$-Se cores had been selectively dissolved with hydrazine. Since the expected peak for Se was not observed, it is believed that the Se cores had been completely removed. 